\title{
RESEARCH OF THE LONG-TERM BEHAVIOUR OF THE PLEIADES BY USING OF PHOTOGRAPHIC PLATES FROM UKRVO DIGITAL ARCHIVE AND BALDONE OBSERVATORY
}

\author{
L.V.Kazantseva ${ }^{1}$, V.M.Andruk ${ }^{2}$, S.V.Shatokhina ${ }^{2}$, Yu.I.Protsyuk ${ }^{3}$, I.Eglitis ${ }^{4}$, M.Eglite ${ }^{4}$ \\ ${ }^{1}$ Astronomical Observatory of Kyiv Shevchenko National University, \\ Observatorna St. 3, Kyiv, 04053, Ukraine, likaz@observ.univ.kiev.ua \\ ${ }^{2}$ Main Astronomical Observatory of National Academy of Sciences, \\ Akad. Zabolotnogo St. 27, Kyiv, 03680, Ukraine, andruk@mao.kiev.ua, \\ svetash@mao.kiev.ua \\ ${ }^{3}$ Research Institute Nikolaev Astronomical Observatory, \\ Observatornaya St. 1, Mykolaiv, 54030, Ukraine,yuri@nao.nikolaev.ua \\ ${ }^{4}$ Baldone Observatory, Institute of Astronomy, University of Latvia \\ Baldones Riekstukalns, Rigas raj., LV 2125, Latvia, ilgmars@latnet.lv
}

\begin{abstract}
The Pleiades Star Cluster (M45) is one of the most studied star clusters in the galaxy. At the same time as part of the Pleiades are many long-period variable stars, including flashing that need further study. Recent work on the processing of photographic images of the Pleiades obtained at different observatory revealed a number of star clusters with large variations in coordinates, the reason you want to understand. Collection of UkrVO unified digital archive contains a number of plates with images of the stars of the Pleiades obtained on different instruments. Processing of this material allows to test out standard programs for digital astronomical images processing. Also we will add information about variable stars and will improve the coordinates and proper motions of cluster stars. Selected material covers the period $1909-1999$ years. The plates have different margins, scale, number of stars on images and obtained limiting magnitude. Exactly this diverse set of options lets you to be sure of results.
\end{abstract}

Keywords: virtual observatory tools - astrometry - techniques: data analysis: star cluster - M45.

\section{Introduction}

Pleiades Star Cluster (M45) is one of the most studied star clusters in the Galaxy, but still continues its active research: rotation, inflation, and lithium in the Pleiades (Somers, 2015); search for free-floating planetary-mass objects in the Pleiades (Zapatero Osorio, 2014); empirical isochrones, luminosity, and mass functions of the Pleiades cluster (Bouy, 2015).

The Pleiades became the object of interest for digitization: possible Cyclic Activity of the Pleiades' Star (Hambaryan, 2003); for studying the long-term behaviors of the Pleiades flare stars (Tsvetkov, 2002); search for extrasolar Planets in the Pleiades (Yamamoto, 2013).

We are considering two problems: testing of a comprehensive program digitized images processing (Andruk, 2010) and search for changes in the laws of time astrometric and photometric data for the stars of the Pleiades.

\section{Plates with Pleiades Star Cluster in UkrVO archives and taken for processing}

Now Joint Electronic Archive of UkrVO (Vavilova, 2012) has 339 M45 images in 16 particular archives, which were obtained in the 8 observation points.

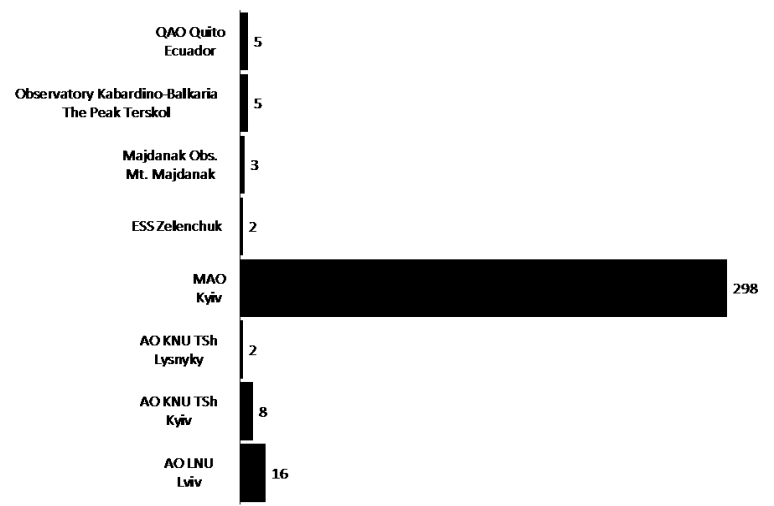

Figure 1: Distribution of observation points data

In addition, we have included in the processing 38 plates of Research Institute "Nikolayev Astronomical Observatory" and 60 plates of Baldone observatory of Institute of Astronomy, University of Latvia.

Photographic plates were scanned using different scanners. Digital images processing was carried out by one and the same comprehensive program. Already wear processed 36 plates for different instruments.
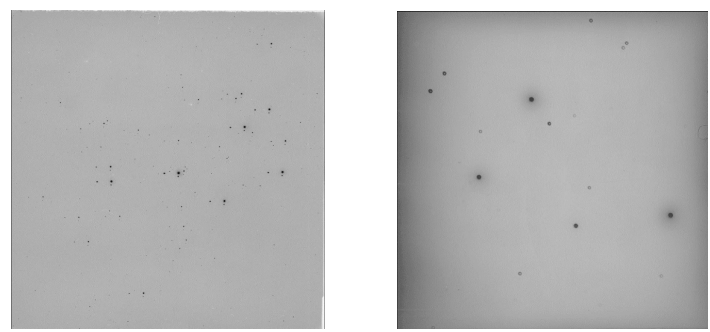

Figure 2: Scan images of plates with different scales. 
Work began recently, made only its initial stage, was processed only $1 / 10$ of the planned material, continues to develop a standard approach to the accurate identification of the stars in a large number of objects in an image, were obtained astrometric and photometric data.

Table 1. Number of plates for each telescope.

$\begin{array}{llllr}\text { Instrument } & \text { AO } & \text { D } & \text { F } & \text { N } \\ \text { Zeiss Zonal Astrograph } & \text { NAO } & 0,1 & 2,0 & 10 \\ \text { Zeiss-600 } & \text { Terskol } & 0,6 & 7,5 & 4 \\ \text { Double Astrograph Repsold } & \text { AOKNU } & 0,2 & 4,3 & 2 \\ \text { Double Long Focus Astrgraph } & \text { MAO } & 0,4 & 5,5 & 9 \\ \text { Double Wide Angle Astrograph } & \text { MAO } & 0,4 & 2,0 & 3 \\ \text { Baldone Schmidt Telescope } & \text { BAO } & 1,2 & 2,4 & 4 \\ \text { Three-Camera Astrograph } & \text { MAO } & 0,1 & 1,7 & 2 \\ \text { Unknown } & \text { AOKNU } & & & 2 \\ - & & & & \end{array}$

\section{Preliminary results}

The scans processing was carried out using MIDAS/ROMAFOT software package, with the individual flat-field calculated for each plate separately (Andruk, 2005; Andruk, 2014).
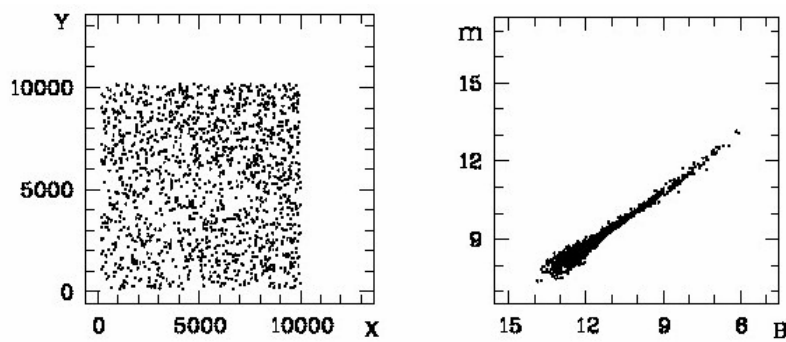

Figure 3: Digital image and the dependence of the magnitude for plate N0078.

The processing of digital images of various sources has been described repeatedly (Kazantseva, 2015; Protsyuk, 2014). We used the standard method of complex processing and compared the results virtually the same region of the sky for the various epochs and observation conditions.

Table 2. The internal accuracy for all objects on the coordinates.

$\begin{array}{lll}\text { Instrument } & \begin{array}{l}\text { mean } \\ \text { rmsRA }\end{array} & \begin{array}{l}\text { mean } \\ \text { rmsDE }\end{array} \\ \text { Zeiss Zonal Astrograph } & 0,135^{\prime \prime} & 0,150^{\prime \prime} \\ \text { Zeiss-600 } & 0,283 & 0,090 \\ \text { Double Astrograph Repsold } & 0,089 & 0,093 \\ \text { Double Long Focus Astrograph } & 0,056 & 0,050 \\ \text { Double Wide Angle Astrograph } & 0,096 & 0,087 \\ \text { Baldone Schmidt Telescope } & 0,129 & 0,108 \\ \text { Three-Camera Astrograph } & 0,092 & 0,087 \\ \text { Unknown } & 0,541 & 0,493\end{array}$
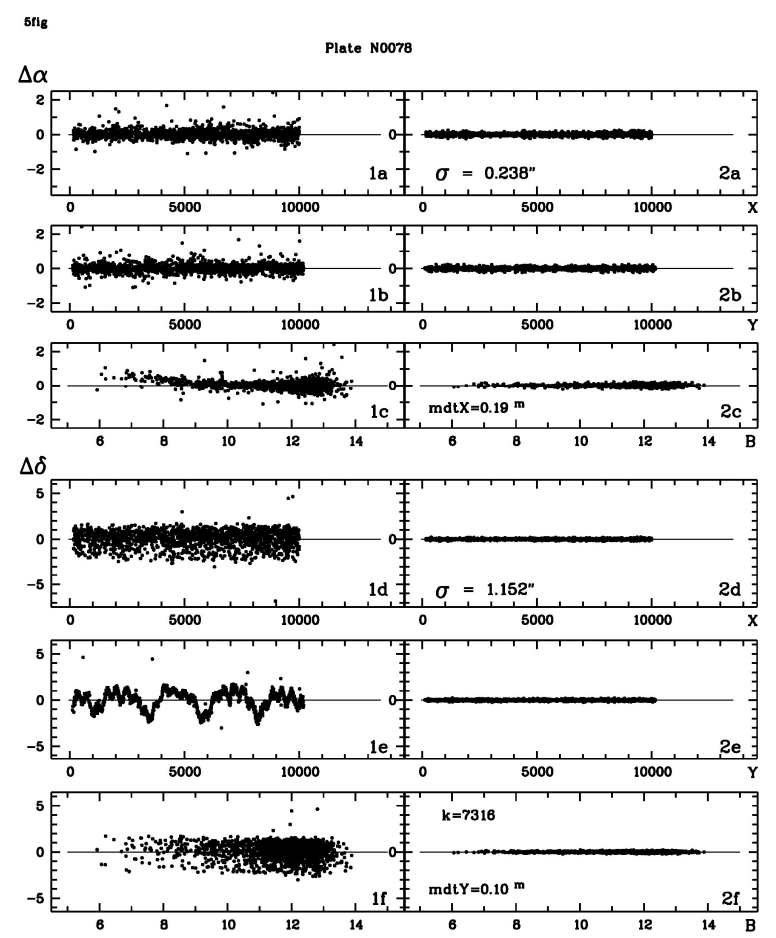

Figure 4: The residual differences in coordinates after the program corrections for plate N0078.

Preliminary analysis of the material obtained allows us to make some conclusions:

- Standard image processing of brightest stars requires improvement.

- Photometric data require additional account information (filters, emulsion, exposure time, etc.).

- Preparation of O-C coordinates on different epochs of observations demonstrate the manifestation of his own movements, which are known for certain stars reach 21 mas/year for RA and 47 mas/year Dec.

\section{References}

Andruk V.M. et al.: 2010, Kinem. Phys. Cel. Bodies, 26, N3, 75.

Andruk V.M. et al.: 2005, Kinem. Phys. Cel. Bodies, 21, N5, 396.

Andruk V.M. et.al.: 2014, Odessa Astron. Publ., 27/1, 53. Bouy H. at al.: 2015, $A \& A, \mathbf{5 7 7}$, id. A148, 17.

Hambaryan V. et al.: 2012, PASRB, 11, 259.

Kazantseva L.V. et al.: 2015, Kinem. Phys. Cel. Bodies, 31, N1, 58.

Protsyuk Yu.I. et al.: 2014, Odessa Astron. Publ., 27/1, 59.

Protsyuk Yu.I. et al.: 2014, Odessa Astron. Publ., 27/1, 61.

Somers G. et al.: 2015, MNRAS, 449, N 4, 4131.

Tsvetkov M. et al.: 2005, Kinem. Phys. Cel. Bodies, Supl., 5, 567.

Vavilova I.B. et al.: 2012, Kinem. Phys. Cel. Bodies, 28, N2, 85 .

Yamamoto K. et al.: 2013, $P A S J, \mathbf{6 5}, \mathbf{N} 4,19$

Zapatero Osorio M. R. et al.: 2014, $A \& A$, 568, id A77, 16. 